L'HOMME L'Homme

171-172 | 2004

Musique et anthropologie

\title{
Expérimenter en ethnomusicologie
}

\section{Nathalie Fernando}

\section{OpenEdition}

Journals

Édition électronique

URL : http://journals.openedition.org//homme/24920

DOI : 10.4000//homme.24920

ISSN : 1953-8103

\section{Éditeur}

Éditions de l'EHESS

\section{Édition imprimée}

Date de publication : 1 décembre 2004

Pagination : 284-302

ISSN : 0439-4216

\section{Référence électronique}

Nathalie Fernando, «Expérimenter en ethnomusicologie », L'Homme [En ligne], 171-172 | 2004, mis en ligne le 01 janvier 2006, consulté le 10 décembre 2020. URL : http://journals.openedition.org//homme/ 24920 ; DOI : https://doi.org/10.4000//homme.24920 


\title{
Expérimenter en ethnomusicologie
}

\author{
Nathalie Fernando
}

$\mathrm{D}$

ANS SON ARTICLE "Enquête d'ethnomusicologie " paru en 1968, Gilbert Rouget (1968) rappelait ce que l'ethnomusicologie devait, en tant que discipline, aux progrès technologiques. Il évoquait le magnétophone à cassettes ou à bandes, les caméras et les possibilités qu'offraient ces appareils pour recueillir et conserver fidèlement tout patrimoine musical. Dans les années qui suivirent, de nouvelles méthodes d'investigation et de nouveaux appareils de mesure furent mis au point, qui permirent non plus seulement d'enregistrer une musique ou de filmer une chorégraphie mais de transcrire des polyphonies complexes et d'accéder ainsi à la connaissance précise des processus compositionnels mis en ouvre. Nous faisons allusion ici à l'utilisation de l'enregistrement en re-recording (Arom 1976) puis en multipistes (Marandola 1999) - techniques qui, toutes deux, permettent d'isoler les voix d'une polyphonie sans pour autant en perdre leur mode d'imbrication. Depuis, l'informatique a ouvert d'autres perspectives en facilitant l'analyse du langage musical et surtout la manipulation des paramètres du son tels que le timbre ou la hauteur.

La possibilité est dès lors offerte au chercheur d'étudier plus aisément les composantes des formes musicales - en délimitant les contours des cellules mélodiques et rythmiques, en cernant leurs modalités de superposition ou en évaluant leur degré de variation au cours de l'exécution. Ce faisant, il s'aperçoit que, dans nombre de cas, l'analyse de la musique qu'il recueille ne révèle qu'une partie du fonctionnement du système musical et ne peut suffire à comprendre certains phénomènes. En effet, si l'on se réfere par exemple aux musiques d'Afrique centrale, l'analyse de la performance permet de décrire les modalités de variations d'une cellule mélodique ou rythmique, mais ne peut aboutir à la connaissance des règles qui permettent cette transformation ou à la mise au jour de la cellule "mère " à

Je remercie chaleureusement Frank Alvarez-Péreyre et Simha Arom pour leurs relectures attentives. Toutes les figures concernant cet article sont renvoyées en annexe, p. 298. 
partir de laquelle elle est effectuée. De même, on ne peut que constater l'instabilité des systèmes scalaires sans pouvoir expliquer, à ce stade de l'analyse, les raisons qui engendrent des fluctuations de hauteur. Enfin, décrypter les processus compositionnels à partir de la seule exécution ne permet pas de comprendre pourquoi deux entités musicales paraissent différentes à l'observateur alors qu'elles sont parfois reconnues par les autochtones comme une seule et même pièce.

Le chercheur doit par conséquent envisager de dépasser le stade de l'analyse immanente pour se concentrer sur les aspects cognitifs de la production musicale. Cela le conduit nécessairement à se confronter à des problématiques plus abstraites et à des situations d'enquête d'autant plus délicates et difficiles qu'elles se déroulent au sein de cultures où le nombre de termes techniques renvoyant à la musique est peu élevé et où l'on constate, au fil de l'enquête, l'absence quasi systématique de verbalisation de la théorie musicale. Se pose alors la question de savoir quelle méthode appliquer et quels outils utiliser pour y parvenir.

D'autres sciences - celles dites "dures »- ont déjà répondu à cette question en élaborant des modalités d'investigation fiables qui ont recours à l'expérimentation. Les principes qui régissent ce type de procédure n’ont pas échappé à des ethnomusicologues tels que Simha Arom, lequel s'en est inspiré pour mettre au point des enquêtes interactives de nature expérimentale lors de ses travaux sur la modélisation des références mentales - travaux qui portaient dans les années 1980-1990 tant sur la forme des pièces que sur les systèmes d'accordage de xylophones centrafricains (Arom 1985, 1998).

C'est ce mode d'investigation que nous souhaitons évoquer ici en valorisant l'apport, sur le plan méthodologique, non pas des nouvelles technologies en tant que telles (qui ont grandement évolué depuis dix ans), mais des expérimentations qu'elles permettent d'effectuer et dont peuvent bénéficier, en premier lieu, les recherches pratiquées dans le domaine de l'anthropologie cognitive.

Précisons que les lignes qui suivent ne sont pas le fruit de considérations abstraites sur l'expérimentation ; elles se réferent à un travail portant sur les systèmes scalaires dans les polyphonies vocales des Pygmées Bedzan du Cameroun, lequel servira de support à notre réflexion et d'exemple à la démonstration ${ }^{1}$.

\section{Les conditions de l'expérimentation}

Tout d'abord, l'expérimentation telle qu'elle est pratiquée en ethnomusicologie est une procédure qui n'intervient qu'après les démarches habituelles, à savoir l'enquête verbale, l'observation et la collecte d'enregistrements. Elle n'exclut nullement le travail d'analyse du répertoire dont elle dépend; au contraire, celui-ci

1. Cette recherche s'est effectuée dans le cadre de l'Action concertée incitative cognitique du ministère de la Recherche, projet A 108 : "Conception et perception des échelles musicales dans les cultures de tradition orale : le cas des Pygmées Bedzan et des Ouldémé du Cameroun ». L'équipe de recherche se composait d'une part d'ethnomusicologues du LMS, unité mixte 8099 du CNRS (Simha Arom - directeur du projet -, Nathalie Fernando, Fabrice Marandola), d'autre part d'acousticiens (René Caussé, Claire Ségoufin, Christophe Vergez) et d'un psycho-acousticien (Daniel Pressnitzer) de l'Ircam. 
révèle si l'expérimentation s'avère ou non nécessaire et, le cas échéant, en détermine les modalités.

Comme dans bien d'autres domaines, le chercheur doit alors manipuler un matériau dont il ne connaît pas encore toutes les propriétés. Dans un premier temps, sa démarche consiste à observer, analyser, forger des hypothèses sur le fonctionnement de ce matériau, sur les formes qu'il est susceptible de revêtir, ou encore, sur ses capacités de transformation par rapport à un contexte donné.

L'expérimentation lui permettra de se focaliser parfois sur des objets infiniment petits qu'il deviendra alors possible d'isoler - ou plutôt de distinguer - au sein de leur environnement. Dans le cas des échelles musicales d'Afrique centrale par exemple, le chercheur ne détache pas l'objet de son contexte habituel mais agit sur certains des paramètres avec lesquels celui-ci se trouve combiné, de façon à neutraliser et à éliminer ainsi les données susceptibles de brouiller le processus de déductions qui fait suite à l'analyse des données.

L'application et, le cas échéant, la mise au point d'un protocole expérimental ne doivent cependant pas être systématiques. Il convient de se demander dans quel contexte il est nécessaire de passer par l'expérimentation : quand, face à quelles musiques et suivant quelles problématiques? Et pour quoi, c'est-à-dire pour accéder à quel type de données?

Par la suite, il s'agit de déterminer comment procéder, en d'autres termes se demander en quoi consistera l'expérimentation, quels seront les outils et les protocoles qui devront être mis en œuvre ? Souvent chaque problématique nécessite de développer un nouvel outil adapté tant au contexte qu'à l'objet.

Quelles que soient les procédures imaginées par le chercheur, elles devront nécessairement reposer sur la collaboration active des musiciens. Expérimenter dans le domaine musical exige en effet un dialogue et une interactivité permanentes entre musiciens et chercheur, échange sans lequel l'expérimentation ne pourrait se poursuivre une fois passée la première étape de la procédure. Nous le verrons, ce sont les réactions des musiciens qui conditionnent les déductions que fera le chercheur - et donc les nouvelles hypothèses qu'il élaborera après chaque opération - et c'est en fonction des réponses fournies par les musiciens que de telles hypothèses pourront ou non être validées. Car l'objectif de toute expérimentation - on le sait - ne consiste pas seulement à disséquer l'objet en question mais à le mettre à l'épreuve du corps d'hypothèses que le chercheur a élaborées ; il s'agit de tester la malléabilité et les limites de ses formes pour en connaître "l'élasticité » et les points de rupture, afin de dégager son mode de fonctionnement.

\section{Quand et pourquoi expérimenter?}

Sur le terrain, l'expérimentation s'impose d'elle-même dès lors que les problématiques qui se présentent ne peuvent être résolues en utilisant des méthodes plus traditionnelles. Lorsque le chercheur confronte les données qu'il recueille grâce à l'enquête, aux enregistrements, à l'observation minutieuse autrement dit qu'il croise ce que les musiciens lui disent à propos de leurs pra- 
tiques avec l'analyse qu'il en fait - et que cette opération devient insuffisante pour comprendre le fonctionnement du système musical dans son ensemble y compris son articulation avec le contexte socioreligieux -, il lui faut élaborer des outils qui lui permettent de progresser dans sa recherche. Dans la plupart des cas, cela revient à tenter de passer de l'autre côté du miroir pour surmonter l'écueil de la verbalisation, voire franchir la limite que constitue, pour les musiciens, la reconnaissance explicite des éléments qui composent leur langage musical. De nombreuses enquêtes effectuées dans les cultures de tradition orale en Afrique centrale ont en effet prouvé que tous les objets que les autochtones conceptualisent ne sont pas nécessairement nommés (Lacito 1987), ce qui correspond, pour le chercheur, à un premier niveau d'abstraction qui doit être dépassé. On peut alors imaginer l'ampleur des difficultés que ce dernier rencontre pour aborder des objets qui, apparemment, échappent à la propre conscience des musiciens.

Lorsqu'il s'agit, par exemple, de saisir comment fonctionne le système d'opposition des hauteurs - sujet qui nous préoccupe ici -, l'ethnomusicologue s'aperçoit rapidement que l'échelle musicale entre directement dans la catégorie "des objets qui échappent à la conscience des musiciens». Car bien qu'elle constitue un outil indispensable à la création de toute forme musicale, l'échelle reste pour eux un élément abstrait, inexistant en dehors du contexte de la performance. Si le chercheur peut quant à lui parfaitement la circonscrire en se référant à sa propre culture - où elle forme une entité en tant que telle -, il n'en demeure pas moins que, pour des musiciens qui appartiennent à une autre culture, l'échelle se trouve totalement confondue avec d'autres paramètres de même niveau (rythme, timbre, etc.). C'est pourquoi la verbalisation locale inhérente à cet objet n'existe pas, ce qui élimine d'emblée la possibilité de travailler en ayant uniquement recours à une enquête de type traditionnel.

Par ailleurs, la problématique que posent au chercheur les musiques d'Afrique centrale renvoie sans cesse à ce phénomène: les musiciens offrent toujours plusieurs versions d'un même objet. Cela implique que chaque réalisation d'une pièce ne révèle pas les mêmes qualités formelles que la précédente. En comparant seulement les traits constitutifs qui sont perceptibles au niveau de la matérialisation de l'objet, il est parfois même impossible de saisir une quelconque similitude entre diverses versions. C'est généralement le cas lorsque les autochtones exécutent une même pièce avec des formations instrumentales différentes, ou qu'ils l'adaptent d'une formation vocale à une formation instrumentale. La forme acoustique de la pièce change alors à un point tel que le résultat sonore devient difficilement identifiable pour des membres extérieurs à la communauté (Arom $\&$ Fernando 2002). Enfin, si on s'attache à observer uniquement le paramètre de la hauteur, on constate qu'une même courbe mélodique peut faire l'objet de plusieurs variantes. Autrement dit, les hauteurs produites, et par conséquent les intervalles qui séparent chaque degré, ne sont jamais les mêmes d'une version à l'autre. Parfois même, ils different - dans la musique vocale notamment - au sein d'une même performance. 
Néanmoins, toutes les versions entrent dans la même catégorie émique (Pike 1964, 1982, 1990; Roulet 1974) puisqu'elles portent le même nom et sont considérées par les membres de la communauté comme culturellement équivalentes. Ces derniers affirment en effet sans hésiter qu'il s'agit de "la même chose ", ce qui sous-entend que les multiples versions que le chercheur distingue recouvrent pour eux une forme similaire et une fonction identique.

On en déduit que chaque version détient au regard des autres ce que Ludwig Wittgenstein (1961) nomme un "air de famille", même si celui-ci n'est pas immédiatement intelligible pour un auditeur non averti. On peut également supposer que, au niveau de la performance de la pièce, cet "air de famille» concerne tant la forme que la fonction des éléments qui la composent, car si la matérialisation d'un même objet musical admet un certain nombre de distorsions d'une fois sur l'autre, c'est que les critères de forme et de fonction entrent conjointement dans les processus autochtones d'identification et de catégorisation des formes. Enfin, cet "air de famille» doit vraisemblablement pouvoir être défini plus précisément à un niveau plus abstrait, celui de la conception mentale de la structure musicale de la pièce.

\section{Nature de l'objet recherché}

Les caractéristiques que nous venons de décrire montrent que nous avons affaire à un système particulièrement flexible dont le fonctionnement repose sur un certain nombre de propriétés. Parmi celles-ci, on compte la variabilité des associations entre les différents éléments qui composent les formes que le système permet de produire, la transformation mutuelle et réciproque de la forme même de chacun de ses éléments et leur équilibre formel réciproque, afin que leur juxtaposition ou leur association puisse répondre à une norme globale.

L'objet qui correspond à cet "air de famille» - et dont on cherche à définir la structure et les contours pour comprendre les phénomènes qui se produisent ne sera sans doute jamais présent en tant que tel au sein d'une seule performance, mais il sera implicitement véhiculé à travers toutes les formes musicales qui en constituent autant d'actualisations. Il se situe donc en amont de la matérialisation que propose le musicien, et on peut raisonnablement émettre l'hypothèse que ses propriétés opèrent également en amont de la forme à laquelle se réfere le musicien au moment même où il passe à l'action.

Plus que des structures formelles, il convient alors de définir les règles et les processus qui permettent de parvenir à l'élaboration d'une forme que les autochtones considèrent comme adéquate, en d'autres termes culturellement acceptable. Il faut déterminer, d'une part, de quelle nature sont les " outils » qui leur servent à concevoir mentalement les formes qu'ils exécutent et, d'autre part, en vertu de quels principes chaque individu peut mettre en ouvre sa propre compétence à exécuter une forme dans un contexte donné. Enfin, il s'agit de découvrir au sein de quel type de système un musicien puise la capacité de créer des formes toujours nouvelles pouvant toutes correspondre à un seul et même objet. 
L'objectif est donc de modéliser non pas une forme en tant que telle, mais plutôt le mode de fonctionnement d'un système, c'est-à-dire les principes d'élaboration qui conditionnent la structure des formes. Le modèle que l'on recherche fait figure ici de prototype abstrait que l'on ne peut parvenir à définir qu'à partir des propriétés typiques des formes issues de la catégorie qu'il caractérise. La principale difficulté tient au fait que celles-ci ne sont pas nécessairement identiques entre elles, qu'aucune ne représente parfaitement ce modèle et que toutes peuvent témoigner d'un trait, de deux traits, voire d'aucun trait commun, si on les compare terme à terme.

Cependant, si ces formes ne partagent pas de propriétés sensibles, elles ont en commun, d'une part, de répondre positivement aux critères auxquels est subordonnée l'acceptabilité d'une forme par les membres d'une communauté et, d'autre part, d'être issues d'un système qui, potentiellement, permet de les engendrer toutes et de les considérer comme culturellement équivalentes. Le prototype que l'on recherche se définit plus en termes de potentialité et de processus qu'en terme de simple calibrage. Le modèle visé ne correspond donc pas à une forme fixe, mais à un réseau dynamique de possibilités.

\section{Comment expérimenter?}

Nous l'avons vu, les multiples matérialisations d'un même objet - et les propriétés qui s'en dégagent - conduisent l'ethnomusicologue à s'intéresser non plus uniquement aux réalités observables et à l'analyse de leurs régularités, mais à aller au-delà de ce qui est immédiatement préhensible et des qualité immanentes de la forme produite, afin de découvrir les potentialités et les modalités de fonctionnement d'un système.

Nous nous référerons à Jean Molino (1975) pour dire qu’il s'agit ici de prendre en compte le niveau poïétique (c'est-à-dire celui de la conception de la forme), le niveau neutre (celui de la réalisation qui correspond à la forme " audible » et qui va permettre, grâce à l'expérimentation, d'accéder au niveau précédent), enfin le nivean esthésique (celui de la perception de l'objet). Le principe heuristique consiste à dégager les propriétés de telle ou telle forme à partir de leur actualisation - niveau neutre -, avant d'émettre des hypothèses sur la façon dont ces formes sont conçues et perçues.

C'est en gardant à l'esprit ces trois niveaux que le chercheur doit penser la problématique et élaborer les protocoles expérimentaux. Ces derniers doivent permettre de recueillir et d'analyser les formes qui apparaissent au niveau neutre afin d'élaborer des hypothèses sur les modes de fonctionnement qui régissent leur conception. Ils doivent également donner au chercheur la possibilité de tester ces hypothèses en créant des formes musicales analogues à celles produites par les musiciens, mais dont les paramètres étudiés auront fait l'objet de modifications. Autrement dit, le chercheur doit pouvoir créer lui-même les formes qui l'autorisent à vérifier, au niveau esthésique, la validité de ses hypothèses, en appliquant ces dernières au niveau neutre afin de les soumettre aux musiciens. Enfin, les pro- 
tocoles expérimentaux doivent reposer sur l'analyse de la réaction des musiciens auxquels ces nouvelles formes auront été soumises. En validant ou non les formes ainsi imaginées, ces musiciens confirmeront ou infirmeront les hypothèses que le chercheur émet sur les critères qui président à leur reconnaissance et à leur catégorisation locales. En d'autres termes, c'est à partir de ce que les autochtones pourront dire de ce qu'ils perçoivent que seront 1) déterminées les caractéristiques qui leur ont permis de classer telle ou telle forme dans telle ou telle catégorie, et 2) mis au jour les critères de catégorisation qui sont les leurs.

Il s'agit donc pour le chercheur de partir du niveau neutre, celui qui correspond aux multiples réalisations d'une même pièce, pour élaborer des hypothèses relatives aux principes sur lesquels repose leur conception - au niveau poïétique -, puis de rendre ces hypothèses intelligibles aux musiciens en créant ou en modifiant les formes appartenant au niveau neutre, avant d'étudier - au niveau esthésique - la réaction de ces derniers.

L'ensemble des procédures à développer repose donc sur les principes d'induction et de déduction. Il s'agit d'induire, à partir de l'analyse des données fournies par les musiciens (les différentes réalisations d'une pièce), une forme hypothétique de l'échelle. Cela revient à élaborer des modèles théoriques formels du système scalaire qui doivent pouvoir être matérialisés en les appliquant directement au répertoire, pour être ensuite proposés aux musiciens. Les modèles devront faire l'objet de rectifications progressives en fonction des réactions de l'auditoire et le chercheur devra déduire de nouveaux modèles théoriques qu'il soumettra une nouvelle fois à évaluation. Il en ira ainsi jusqu'à ce que tous les résultats soient validés et "contre-validés". En effet, une fois que l'on pense avoir cerné et défini la nature de ce qu'on recherche, il est essentiel de valider par la négative, c'est-à-dire soumettre aux musiciens des propositions dont on suppose qu'elles vont être refusées. Les procédures mises au point doivent donc permettre de construire des formes qui n'ont encore jamais été réalisées, afin d'estimer leur degré de vraisemblance. Cette étape est indispensable dans la mesure où on imagine que les formes spontanément actualisées par les musiciens répondent nécessairement à la norme que l'on recherche et que, par ailleurs, ces formes ne mettent jamais en œuvre simultanément toutes les limites du système. Valider des formes musicales que les musiciens n'auraient jamais livrées constitue donc un pas considérable vers la compréhension des procédures d'élaboration de celles qui sont les leurs. Une fois encore, seule l'expérimentation peut permettre d'y parvenir car, dans le cas contraire, il faudrait attendre que les musiciens commettent une erreur pour repérer à quel moment - ou à partir de quand - sont franchies les limites du système. Encore faudrait-il être à même de comprendre à quel niveau cette erreur se situe et de quoi elle est faite.

\section{Plus concrètement}

Les données de la problématique et les questions plus théoriques que celle-ci soulève montrent que l'expérimentation peut être envisagée comme un outil 
venant en aide au chercheur pour travailler sur ce qui n'est pas verbalisé mais aussi - c'est sans doute son principal atout - sur ce qui n'est pas verbalisable. Pour illustrer l'apport indéniable de la méthode expérimentale quant à la mise au jour de tels savoirs, nous exposerons brièvement ci-dessous l'essentiel des étapes de la procédure que nous avons testée chez les Pygmées Bedzan - l'objectif étant de comprendre comment les musiciens bedzan conçoivent leur système d'opposition de hauteurs et quelles sont les règles qui sous-tendent les innombrables variantes relevées dans la matérialisation de leurs systèmes scalaires.

La polyphonie vocale des Bedzan se compose de quatre parties distinctes - la voix des "grands hommes", des "jeunes hommes", des "femmes" et des " enfants». Elle est préalablement enregistrée dans un format multipistes, ce qui permet de visualiser sur un écran d'ordinateur le parcours de chaque voix ainsi que son mode d'imbrication avec les trois autres. On peut également isoler, à n'importe quel moment, l'une ou l'autre des voix. Afin de neutraliser les problèmes liés à la surabondance de variations mélodico-rythmiques et pour que ces dernières ne viennent pas interférer avec celles qui concernent le champ de dispersion des degrés, nous demandons aux musiciens de chanter en évitant, autant que faire se peut, les variations. En procédant par analyse spectrale, nous extrayons les hauteurs des fréquences fondamentales avant de les analyser. Une application spécifique - un "patch ${ }^{2}-$, qui a été développée pour analyser les courbes mélodiques ainsi obtenues, livre plusieurs types de données qui nous permettent notamment de quantifier le nombre de fois où tel degré est chanté à telle hauteur sur un laps de temps donné, ou encore son champ de dispersion au sein d'une même voix, mais aussi dans les différentes parties constitutives de la polyphonie.

L'analyse de ces diverses données aboutit à l'élaboration d'hypothèses sur la constitution des échelles musicales. Chacune des hypothèses est alors testée en l'appliquant à la polyphonie originale enregistrée. Ainsi les musiciens entendentils, chantée par des membres de leur propre communauté, une pièce issue de leur répertoire, mais présentée selon plusieurs versions dont chacune propose des modifications scalaires plus ou moins sensibles par rapport à la précédente ${ }^{3}$.

Mettre au point une chaîne d'opérations cohérentes, c'est-à-dire conduire logiquement la succession des hypothèses qu'il faudra soumettre aux musiciens, n'est cependant pas sans difficulté. Tout d'abord, parvenir à faire fonctionner, sur le terrain, un dispositif de ce type, ne signifie pas uniquement répondre directement aux attentes du chercheur ou encore inventer le meilleur moyen de résoudre les problèmes qui se posent; cela implique que les procédures qui seront soumises aux musiciens - ou auxquelles ils seront soumis - demeureront pertinentes dans le contexte culturel qui est le leur. Autrement dit, que les interrogations que le chercheur soumettra aux musiciens et surtout, la façon dont il les soumettra, feront sens pour eux - car ces derniers doivent pouvoir y répondre.

2. Cette application nommée Scala a été réalisée par Fabrice Marandola à partir du logiciel Open Music de l'Ircam.

3. Le lecteur pourra se reporter aux plages \# 34 et \# 35 du CD d'accompagnement qui correspondent respectivement aux quatre voix enregistrées puis modifiées de la pièce $n \tilde{a}$. 
Par ailleurs, mener rigoureusement ce type d'expérimentation exige de veiller à l'application de la même rigueur à chaque étape de la procédure. Or, les écueils sont nombreux. Il faut tout d'abord s'assurer qu'à tout moment de l'expérimentation, les musiciens se focalisent bien sur l'objet que l'on traite. Les propositions doivent être conçues de façon à ce que l'on sache sur quel élément porte leur acceptation ou leur refus, en d'autres termes, quel est l'intervalle qu'ils acceptent ou rejettent (ce qui implique la présence d'un minimum d'inconnues au sein de chaque nouvelle hypothèse). Il faut également être à même d'élaborer suffisamment d'hypothèses et de propositions pour comprendre pourquoi ils acceptent ou refusent. La principale difficulté réside dans la conception même des modèles théoriques puisqu'en modifiant la hauteur d'un degré, on modifie simultanément la grandeur des intervalles qui le séparent des deux degrés qui lui sont conjoints ; on modifie alors deux éléments au moins, ce qui a pour effet de transformer la configuration scalaire de l'échelle, c'est-à-dire la modalité de succession des intervalles, dans son ensemble. Il est impératif de toujours veiller à réaliser une proposition et son contraire, afin de s'assurer que les réponses obtenues permettent d'éliminer toute ambiguïté.

\section{Réactions de l'auditoire}

Introduire ce type d'expérimentation sur le terrain modifie de façon très sensible le caractère habituel de l'enquête. Tous ou presque tous les membres de la communauté sont présents à chacune de nos séances de travail qui se déroulent au milieu du village, à l'ombre de quelques feuilles de palme.

Comme nous l'avons dit plus haut, le chercheur n'a nul besoin de discourir longuement ou de se perdre dans des explications pour introduire la proposition qu'il va soumettre à audition, ce qui semble préserver d'autant la neutralité des échanges. Il fait directement entendre les versions qu'il propose et observe les réactions de l'auditoire. La question et l'écoute ne font qu'un et les protocoles sont conçus de telle sorte que les auditeurs ne puissent se focaliser que sur le paramètre qui fait l'objet des tests. Bien sûr, les musiciens sont mis dans une situation qui ne leur est guère habituelle et, de prime abord, on devine de leur part une crainte d'être testés sur leur propre compétence. Les regards sont méfiants, interrogateurs, les gestes hésitants; les visages manifestent de l'étonnement à l'idée d'une procédure si insolite, et les remarques ne manquent pas. Et on peut observer comment, de la discussion - et de l'interaction qui naît entre les musiciens - se dégage peu à peu un consensus qui révèle progressivement et nécessairement la "norme" que tous se doivent de respecter, même si, au regard de celle-ci, chacun négocie des variantes qui lui sont propres.

On peut constater que l'attitude des musiciens et la façon dont ils réagissent aux propositions sont exactement identiques à celles dont ils font preuve à l'écoute de membres de leur communauté chantant "en direct». Ils sont aussi prompts à manifester leur approbation ou, au contraire, leur mécontentement. On s'aperçoit alors que si la théorie ne peut être explicitée avec des mots, il n’en 
demeure pas moins que l'expérimentation favorise - voire provoque - l'émergence d'un métalangage que le chercheur ne pourrait découvrir avec tout autre forme d'investigation. On ne peut qu'admirer la manière dont les autochtones savent expliquer, malgré l'absence d'un vocabulaire technique, pourquoi ils refusent ou acceptent telle ou telle proposition : "Ce chant est sucré », signifie qu'il est bien réalisé ainsi, ou encore "Non! son pied est déjà en brousse!", sousentendu, "Sa voix s'égare et la mélodie s'éloigne du chemin », bref, «Il chante faux!». Il est hors système.

Plus on progresse dans l'expérimentation et plus on soumet de propositions, plus les musiciens se détendent et finissent par réaliser que celui qui est en difficulté est en face d'eux et non pas l'inverse. Ils comprennent peu à peu qu'ils vont devoir guider les pas de l'ethnomusicologue. Ce sont eux qui vont alors observer ses manœuvres, guetter la moindre modification, évaluer ses progrès. Et c'est le chercheur qui, à son tour, est plongé dans une situation peu habituelle. L'expérimentation le confronte aux difficultés de l'apprentissage; les musiciens vont corriger les fautes qu'il commet par l'intermédiaire de sa "machine", comme il le feraient avec l'un des leurs qui tenterait de comprendre, tout en chantant, les limites du système musical qu'il découvre. Ils vont caricaturer ce qui est mauvais puis rechanter comme cela devrait être, pointer immédiatement un doigt réprobateur à l'écoute d'une erreur ou manifester leur satisfaction à l'audition d'une mélodie correcte. Au fur et à mesure que nous affinons les hypothèses qui leur sont soumises, les réponses deviennent corollairement plus précises : les musiciens procèdent souvent en rechantant le mouvement mélodique qu'ils viennent d'entendre - en insistant sur le degré qu'il convient de changer - et en commentant de la sorte: "Ce son là est trop à terre", si le degré est trop bas, ou encore "La voix est trop grosse ", lorsqu'il s'agit de baisser la hauteur de telle note. Le degré d'acceptabilité d'une hypothèse peut également être jugé au temps de réaction : si la forme globale est acceptable, hormis quelques défauts, la réaction des musiciens se fera plus lente, plus hésitante. Il faudra alors, pour s'assurer de la validité de l'échelle proposée, repasser plusieurs fois chaque voix et cerner l'intervalle qui n'est pas tout à fait bien réalisé. Il apparaît alors que leur capacité de discrimination est extrêmement fine et que leur niveau d'exigence ne transige pas avec la qualité. Il est ainsi émouvant de constater avec quel plaisir, après maintes propositions défectueuses, maintes corrections, les musiciens accueillent avec un sourire complice une proposition adéquate. On perçoit alors dans leur regard une expression qui signifie : «Il a enfin compris!».

Si l'ambiance qui règne alors est celle d'un jeu, elle n'exclut pas pour autant une légère tension et une concentration qui témoignent du sérieux et de l'application que ces maîtres mettent à partager leur savoir-faire. À chaque proposition, les musiciens semblent rechercher dans leur mémoire si la forme qu'on leur propose correspond à des schèmes qu'ils ont construits au cours de leur apprentissage et de l'expérience qu'ils ont faite des catégories cognitives formelles de leur propre culture. Les réponses qu'ils donnent permettent en effet de comprendre qu'ils jaugent le degré de "typicalité » du modèle théorique qu’on leur propose 
en ce qu'ils ne répondent pas simplement par oui ou par non, mais opèrent une classification progressive des formes acceptables : "Celle-ci est très bien ", "Cellelà est bien mais pourrait être mieux" ou encore, "Celle-là n'est pas tout à fait bien même si on peut l'exécuter ainsi ". Ces réponses nous conduisent à penser que les processus qui aboutissent à la formalisation d'une échelle doivent nécessairement respecter un certain équilibre. Il est vraisemblable que le "plus ou moins bien " renvoie au fait que trop de règles n'ont pas été respectées et que l'on approche du point de rupture.

\section{Acquis et atouts d'une telle méthode}

En réalité, les expérimentations ont montré que l'élaboration des échelles musicales bedzan repose sur une rigueur et une souplesse qui nécessitent d'envisager les systèmes scalaires comme de véritables phonologies dont les unités constitutives relèvent de catégories conceptuelles définies par un faisceau de contraintes réciproques - contraintes qui s'exercent au niveau de "blocs" combinant plusieurs intervalles entre eux. Ce modèle explicatif permet de comprendre non seulement l'extraordinaire mobilité des degrés au sein de ces systèmes, mais également comment un même système scalaire peut être réalisé à partir d'une seule grandeur d'intervalle constitutive (échelle de type équi-pentatonique) ou au contraire admettre autant de grandeurs qu'elle compte d'intervalles ${ }^{4}$.

Seule l'expérimentation telle qu'entendue ici a permis de révéler la nature profonde de ce système en passant d'une analyse des éléments constitutifs, de leur structure et de leur combinatoire à une modélisation qui s'attache au fonctionnement même du système et qui est plus à même de rendre compte des processus interagissant entre ces éléments. Les atouts d'une telle procédure d'investigation sont en effet multiples :

- En prenant appui sur le répertoire traditionnel, elle permet de traiter la problématique dans le contexte culturel sous l'aspect d'une performance.

- Le chercheur domine parfaitement les données et contrôle la forme de la performance en maîtrisant un maximum de paramètres.

- Elle procure l'avantage de se focaliser sur un seul paramètre, sans toutefois le désolidariser des autres.

- L'expérimentation permet de dépasser le stade de l'analyse immanente, c'est-à-dire l'opération qui consiste notamment à analyser et à mesurer les formes telles qu'elles sont produites par les musiciens. En matérialisant ses hypothèses de façon culturellement pertinente, mais aussi en créant des formes qui n’ont jamais été produites, le chercheur est ainsi amené à comprendre pas à pas comment est conçu un objet musical en amont de la matérialisation que les musiciens lui en donnent. Il peut indirectement les interroger sur la façon dont ils perçoivent les formes et dégager des hypothèses au sujet des conditions de leur identification ou de classement dans telle ou telle catégorie émique. Autrement dit, l'expérimentation permet de dégager la nature émique des formes et d'élaborer des hypothèses étiques - mais néanmoins vrai-

4. Pour des résultats complets, voir Simha Arom, Nathalie Fernando \& Fabrice Marandola, Journal de l'Esem, Vilnius (à paraître). 
semblables d'un point de vue émique - sur les processus d'élaboration et les modalités de fonctionnement du système qui les engendre.

- Parce qu'elle repose essentiellement sur la validation des hypothèses par les musiciens locaux, l'expérimentation permet de tester les limites de ce système et d'appréhender, de manière rigoureuse, les contraintes imposées par la norme.

- La méthode expérimentale privilégie la neutralité des échanges. Si la procédure est suivie avec rigueur, l'enquête se déroule sans le risque que le chercheur puisse induire une directivité quelconque au sein de ce type de questionnement. Les musiciens ne répondent pas à des questions, ils réagissent à des propositions.

- Enfin, en offrant l'accès au non verbalisé comme au non verbalisable - tout en cernant précisément l'objet de la recherche et sans déstabiliser les musiciens - l'expérimentation constitue sans nul doute le seul moyen de trouver une réponse aux problèmes posés. Les problèmes théoriques ne sont, dès lors, plus abordés par le discours mais à travers les savoir-faire.

Les procédures expérimentales décrites ici mettent en jeu une combinaison de protocoles et de leur application cherchant à révéler, au-delà des structures formelles qui sous-tendent telle ou telle réalisation, la cohérence et la logique culturelles d'un ensemble de conduites implicites. L'expérimentation permet de dépasser le stade de la description des formes perceptibles en se focalisant davantage sur les processus qui en sont à l'origine. Elle permet de cerner, pas à pas, le fonctionnement non plus d'un modèle figé, mais d'un modèle dynamique rendant compte des principes d'organisation de certaines dimensions dont la combinaison est, selon le contexte, à géométrie variable.

Si la finalité du dispositif vise, dans le cas présent, à la compréhension des systèmes scalaires, les enjeux de ce type d'expérimentation sont avant tout d'ordre méthodologique. Le chercheur peut dégager les unités culturelles qui entrent dans la constitution d'un objet et déterminer la nature des formes en fonction de tel ou tel contexte ; il peut éventuellement en prévoir le comportement et comprendre pourquoi le système laisse la liberté à chaque musicien de proposer une version différente de tel ou tel objet. Du même coup, il peut appréhender la distance qui sépare la norme collective des variabilités individuelles qu'elle admet. Il peut enfin émettre des hypothèses sur la nature des représentations mentales des normes et des repères mentaux des musiciens, sur les processus d'élaboration et d'identification des formes, c'est-à-dire sur les principes logiques qui président à leur fonctionnement catégoriel, ou encore sur la façon dont un musicien puise au sein du système la capacité de créer d'autres formes.

On peut alors progresser dans le domaine des sciences cognitives sur la modélisation de systèmes complexes dans la mesure où, d'une part, ce type d'expérimentation vise à la compréhension des relations réciproques qui prévalent entre les différents niveaux à partir desquels l'objet musical peut être appréhendé (ceux de sa conception, de sa matérialisation et de sa perception), d'autre part, il prend en compte les processus de stimulation mémorielle en ce que la conception, la réalisation et l'identification d'une forme résultent de la dialectique permanente 
qui prévaut entre les différents types de mémoire auxquels le musicien fait appel, à savoir la mémoire à long terme et la mémoire à court terme. Enfin, la méthode présentée ici ouvre de nouvelles perspectives quant à la compréhension des modalités de création et d'appréhension des phénomènes musicaux, ainsi qu'aux universaux qui les sous-tendent.

MOTS CLÉS/KEYWORDS: anthropologie cognitive/cognitive anthropology - expérimentation/experimentation - modélisation/computer modelling - échelle musicale/musical scale - Afrique centrale/ Central Africa.

\section{RÉFÉRENCES BIBLIOGRAPHIQUES}

Arom, Simha,

1976 «The Use of Play-Back Techniques in the Study of Oral Polyphonies ", Ethnomusicology 20 (3) : 483-519.

1985 Polyphonies et polyrythmies instrumentales d'Afrique centrale. Structure et méthodologie. Paris, SELAF, 2 vol.

1991 "L'étude des échelles dans les musiques traditionnelles: une approche interactive ", Analyse musicale 2: 21-24.

\section{Arom, Simha \& Nathalie Fernando}

2002 «'ethnomusicologie est-elle condamnée à rester une science molle?", in Observation, analyse, modèle : peut-on parler d'art avec les outils de la science? Les cahiers de l'Ircam, Paris, l'Harmattan : 427-450.

\section{Arom, Simha \& Frédéric Voisin}

"Theory and Technology in African Music ", in R. Stone, ed, The Garland Encyclopedia of World Music, 2. New York, Garland, 1998 : 254-270.

Marandola, Fabrice

1999 "L'apport des nouvelles technologies à l'étude des échelles musicales d'Afrique centrale ", Journal des africanistes 69 (2): 109-119.

Molino, Jean

1975 «Fait musical et sémiologie de la musique ", Musique en jeu, 17 : 37-62.

Nattiez, Jean-Jacques

1987 Musicologie générale et sémiologie. Paris, Éd. Christian Bourgois.

\section{LACITO}

1987 Nommer son milieu naturel. Paris, Éd. du Lacito.

Pike, Kenneth L.

1964 "Towards a theory of the Structure of Human Behavior", in Language in Culture and Society. Dell H. Hymnes, ed., NewYork, London, Harper \& Row : 54-61.

1982 Linguistic Concepts: An Introduction to Tagmemics. Lincoln, University of Nebraska Press.

1990 "On the Emics and Etics of Pike and Harris", in Emics and Etics.

The Insider/Outsider Debate, Thomas N Headland, Kenneth I. Pike and MarvinHarris, eds, Frontiers of Anthropology vol. 7 , Newbury Park, Sage Publications : 28-47.

\section{Rouget, Gilbert}

1968 "L'enquête ethnomusicologique", in Jean Poirier, ed., Ethnologie générale. Paris,Gallimard : 333-348 (( Encyclopédie de la Pléiade»).

\section{Roulet, Eddy}

1974 Linguistique et comportement humain. L'analyse tagmémique de Pike, Neuchâtel, Paris, Delachaux et Niestlé.

\section{Wittgenstein, Ludwig}

1961 Tractatus Logico-Philosophicus; (suivi de) Investigations philosophiques. Paris, Gallimard, 1961. 
Figure I.Transcription des premiers cycles de l'extrait correspondant à la version originale (enregistrée le 10/08/200 I à Mbondé), plage \# 34 du CD. Dans le premier système, les nombres figurant sous chaque note correspondent à leur déviation (en cents) par rapport au système tempéré. I cent $=1 / 100^{\circ}$ de demi-ton.

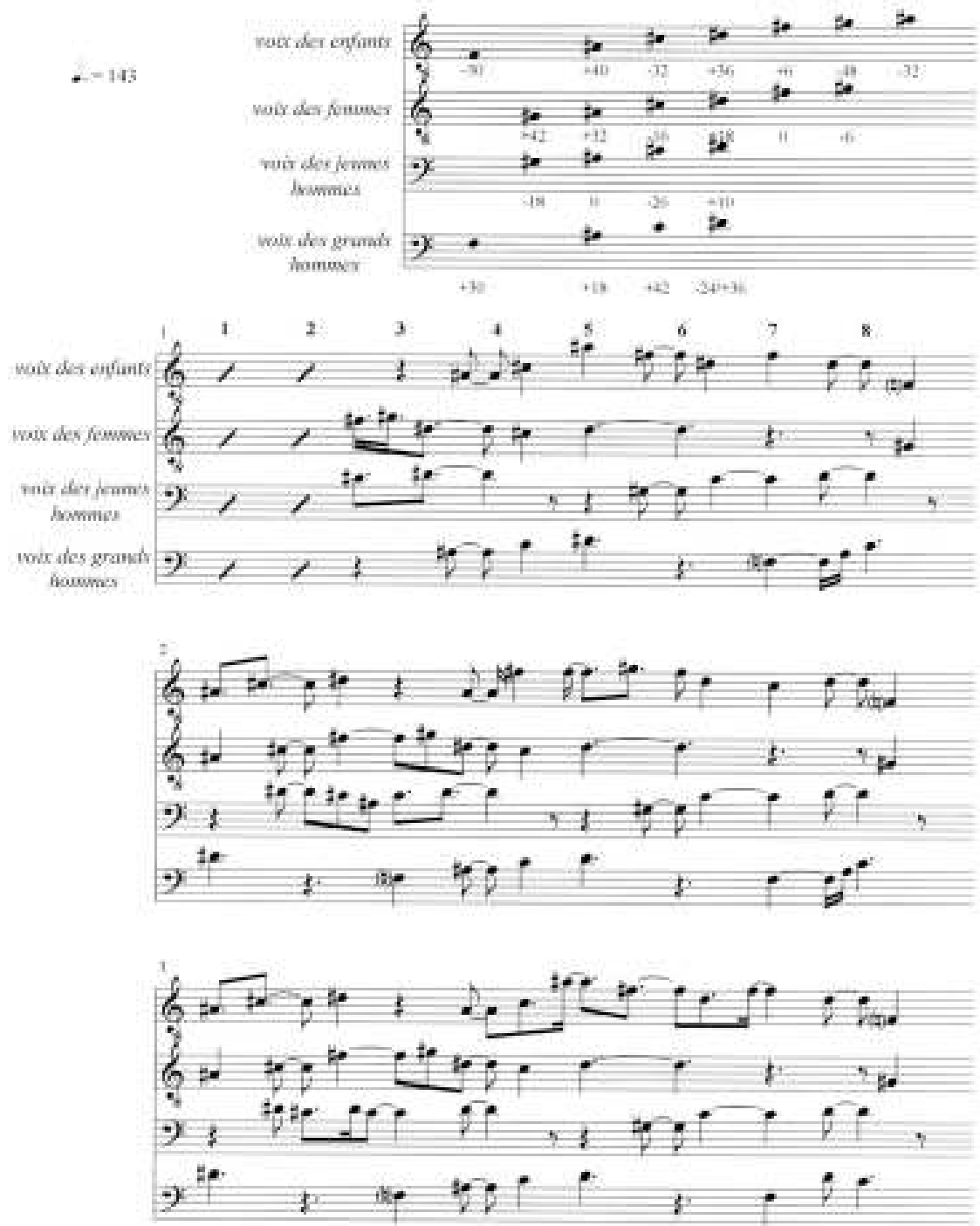



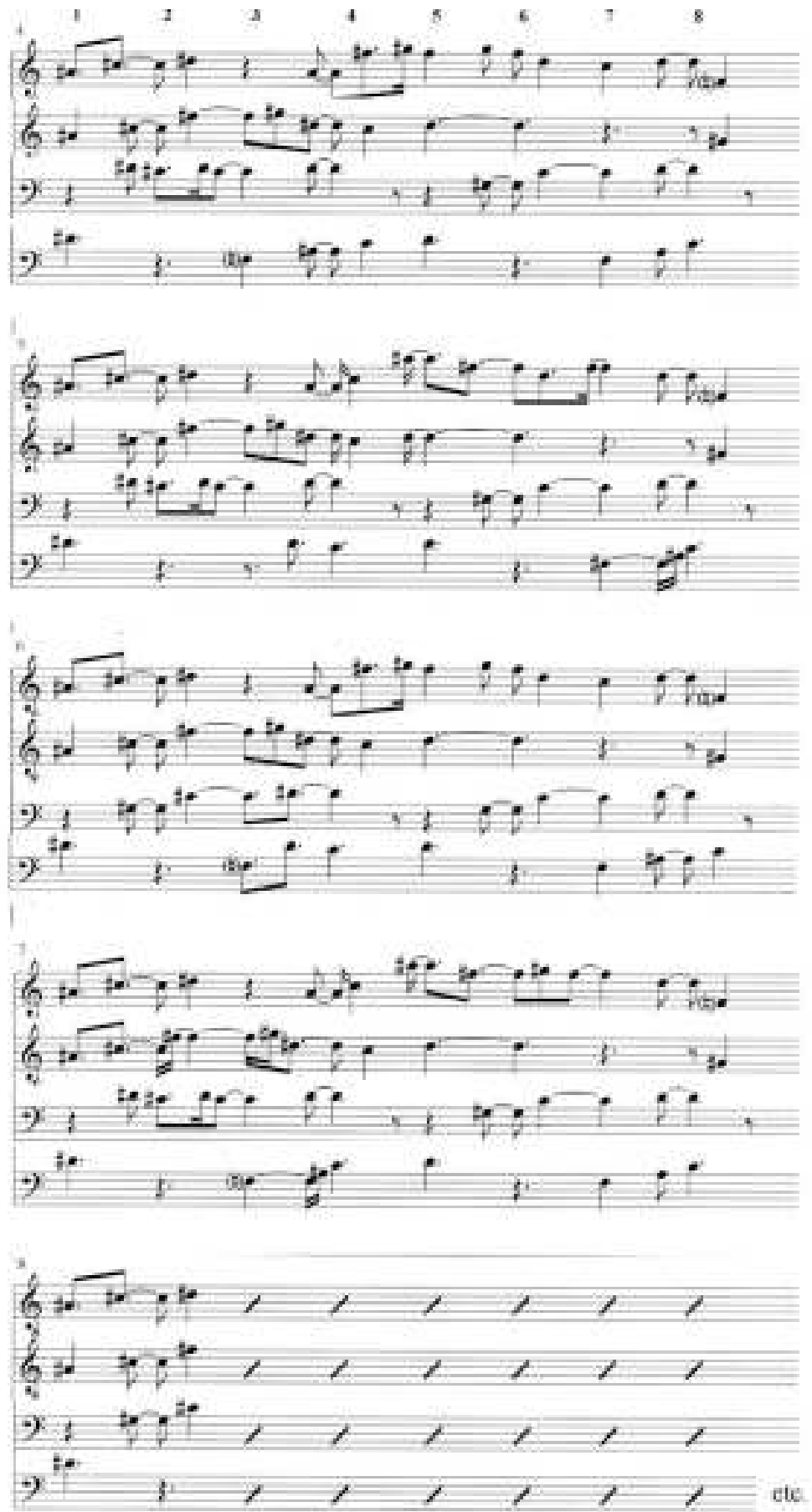


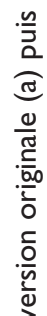

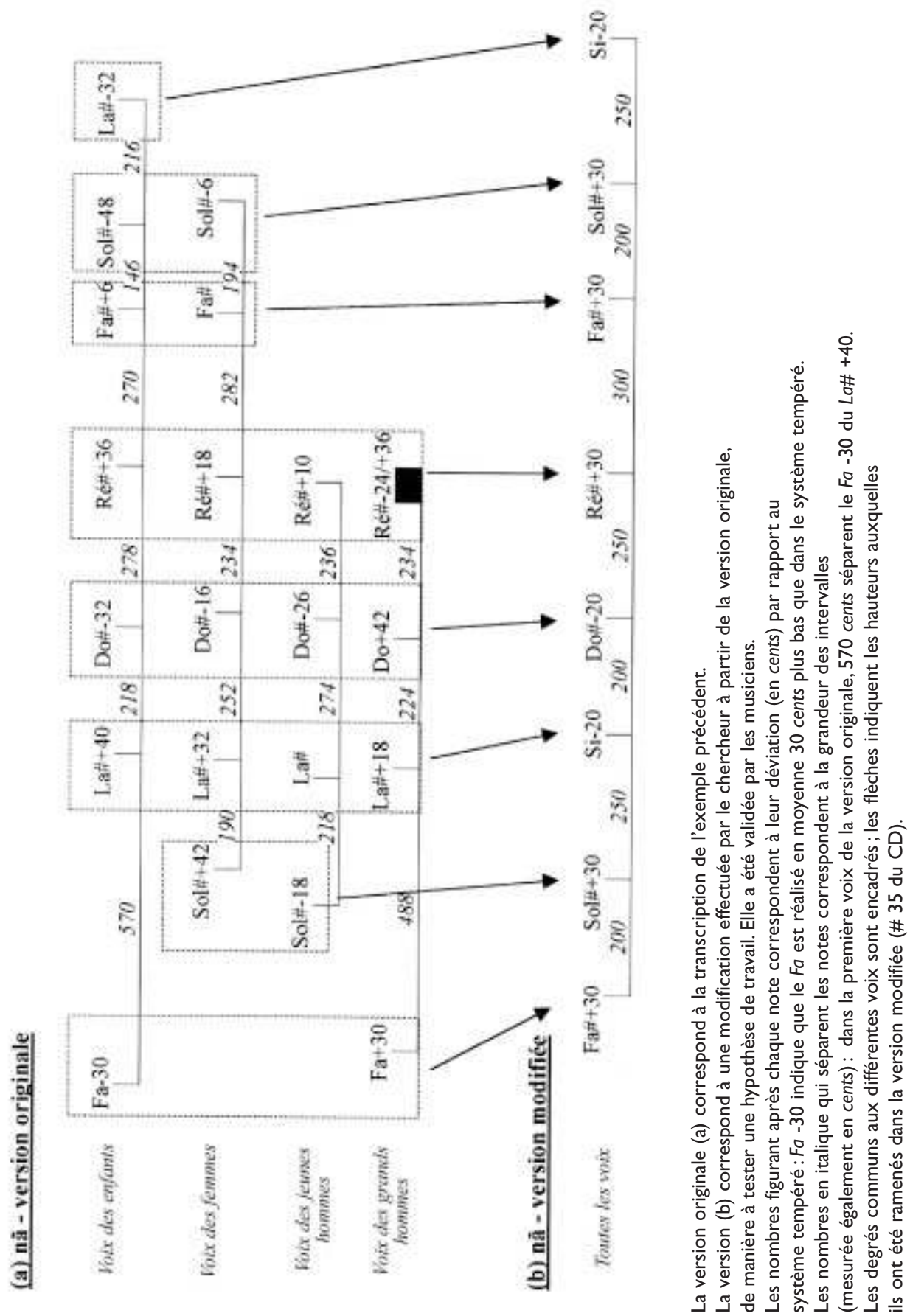


Figure 3. Représentation graphique de l'une des voix de la polyphonie (voix des enfants), permettant de visualiser les transformations qui surviennent entre la version originale et la version modifiée.

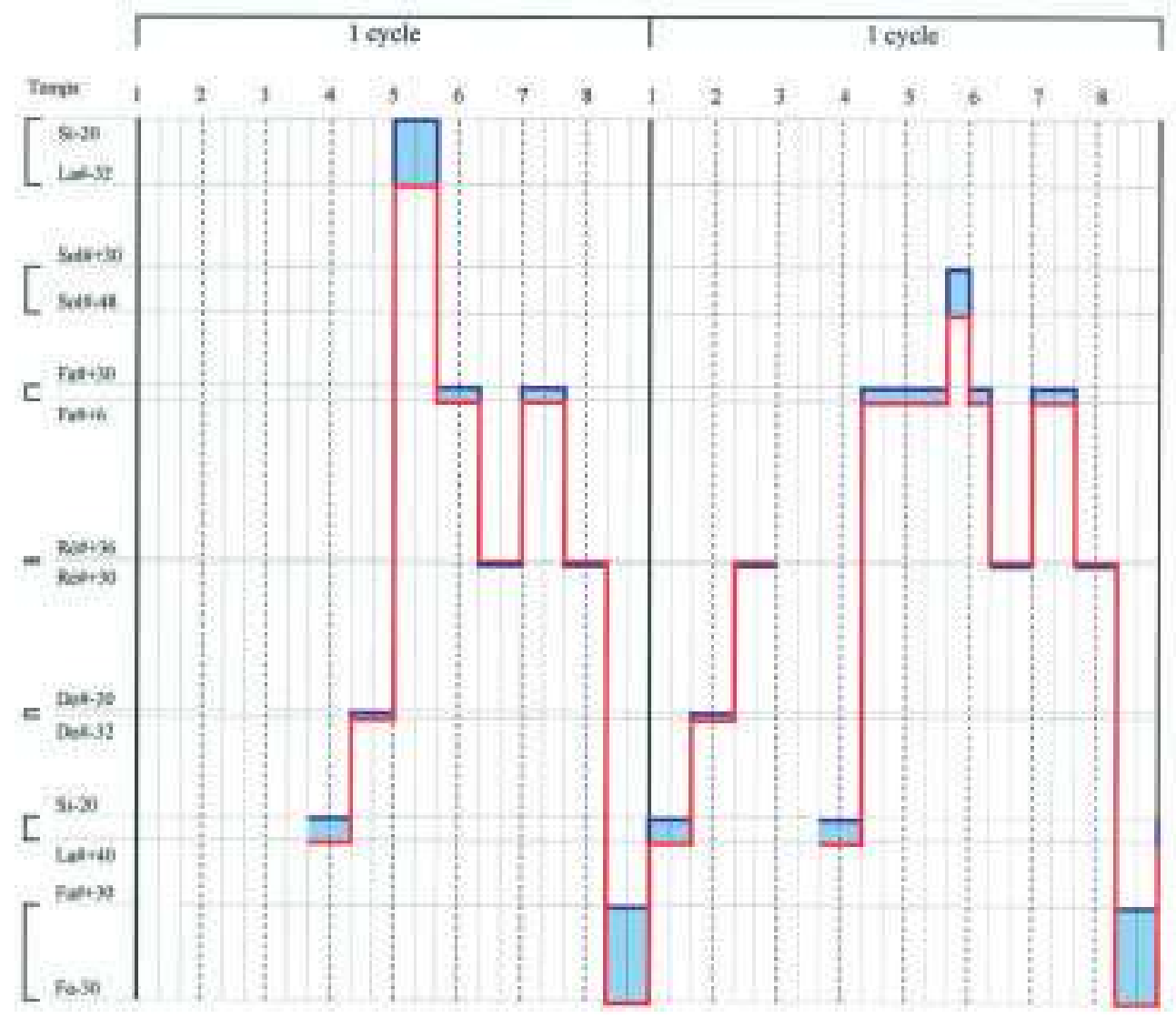

En rouge : version originale.

En bleue: version modifiée.

Le premier temps de cette représentation correspond au premier temps de la transcription sur portée. 
Nathalie Fernando, Expérimenter en ethnomusicologie. - Nombreuses sont les sociétés de tradition orale qui n'explicitent pas leur théorie musicale. C'est pourquoi l'ethnomusicologue peut recourir à une méthode d'approche expérimentale lui permettant d'émettre des hypothèses culturellement plausibles quant aux stratégies cognitives mises en œuvre par les musiciens. Cet article se propose, d'une part, de présenter les récentes avancées en la matière - lesquelles portent sur la modélisation des échelles musicales en Afrique centrale - et d'évoquer, d'autre part, les problèmes de méthode liées à l'expérimentation in situ.
Nathalie Fernando, Experimenting in Ethnomusicology. - Since many societies with oral traditions do not explicitly formulate a theory of music, ethnomusicologists can use a method based on an experimental approach to draw up plausible cultural hypotheses about musicians' cognitive strategies. Recent advances in this field, having to do with the modelization of scales of music in central Africa, are presented ; and the methodological problems of in situ experimentation, discussed. 